\section{Percepção dos usuários e profissionais de saúde sobre atenção básica: comparação entre unidades com e sem saúde da família na Região Centro-Oeste do Brasil}

\author{
Perceptions of primary health care among users \\ and health professionals: a comparison of units \\ with and without family health care in \\ Central-West Brazil
}

Cornelis Johannes van Stralen 1

Soraya Almeida Belisário 1

Terezinha Berenice de Sousa van Stralen 1

Ângela Maria Dayrell de Lima 1

Alice Werneck Massote 1

Cláudia di Lorenzo Oliveira 1

\section{Introdução}

\footnotetext{
${ }^{1}$ Faculdade de Medicina, Universidade Federal de Minas Gerais, Belo Horizonte, Brasil.

Correspondência C. J. van Stralen Núcleo de Estudos em Saúde Coletiva, Faculdade de Medicina, Universidade Federal de Minas Gerais. Av. Alfredo Balena 190, Belo Horizonte, $M G$ 30130-100, Brasil. stralen@medicina.ufmg.br
}

\section{Abstract}

This study analyzes perceptions of performance by primary health care facilities with and without the Family Health Program in municipalities with more than 100,000 inhabitants. Questionnaires from the Primary Care Assessment Tool developed by John Hopkins University and adapted to Brazil, contemplating eight dimensions of primary health care, were applied to users and professionals from a sample of 36 family health care facilities and 28 traditional primary care facilities. Thirty health professionals with university education, 207 with secondary education, 490 adult users, and 133 family members answered the questionnaires. The overall result did not show significant differences between perceptions of family health care facilities as compared to traditional primary health care facilities, but perceptions of health professionals were consistently more favorable than those of users. Comparing the scores for each dimension, family health care facilities always scored better (with the exception of level of access), but the difference in scores between facilities with and without the Family Health Strategy was only statistically significant for all three categories of respondents in relation to the items "family focus" and "community orientation".

Primary Health Care; Family Health; Evaluation; Perception
De acordo com a declaração da Conferência de Alma-Ata de 1978 sobre Atenção Primária, "Cuidados primários de saúde são cuidados essenciais de saúde baseados em métodos e tecnologias práticas, cientificamente bem fundamentadas $e$ socialmente aceitáveis, colocadas ao alcance universal de indivíduos e famílias da comunidade, mediante sua plena participação e a um custo que a comunidade e o país possam manter em cada fase de seu desenvolvimento, no espírito de autoconfiança e automedicação. Fazem parte integrante tanto do sistema de saúde do país, do qual constituem a função central e o foco principal, quanto do desenvolvimento social e econômico global da comunidade. Representam o primeiro nível de contato dos indivíduos, da família e da comunidade com o sistema nacional de saúde, pelo qual os cuidados de saúde são levados o mais proximamente possível aos lugares onde pessoas vivem e trabalham" 1 (p. 1).

O Ministério da Saúde do Brasil não enviou delegados para essa conferência, mas já estava investindo na atenção primária por meio de programas de extensão da cobertura de serviços de saúde, entre os quais se destacava o Programa de Interiorização de Ações de Saúde e Saneamento (PIASS). O PIASS visava à implementação de serviços básicos de caráter permanente, preconizando ampla utilização de pessoal auxiliar, ênfase na prevenção de doenças, desenvolvimento 
de ações de saúde de baixo custo e alta eficácia em caráter permanente, organização de sistemas regionalizados e hierarquizados, e ampla participação comunitária 2. José Carlos Seixas, então Secretário Geral do Ministério da Saúde destacou no seu pronunciamento na VI Conferência Nacional de Saúde (1977) que "O PIASS não é mais um programa de saúde a ser desenvolvido; é uma metodologia de trabalho, para a implantação de uma estrutura permanente de Saúde Pública, a nível da população necessitada de bens e serviços básicos de saúde - saneamento. Não é, pois, algo que se justapõe ao já existente, mas é algo que deva modificar de forma permanente o que existe" 3 (p. 22).

Passaram-se muitos anos, mas permanece o desafio de organizar a atenção primária ou atenção básica não meramente como estratégia de controle de gastos mas, principalmente, como componente crítico do sistema de saúde. Atualmente, a principal estratégia de implementação e organização da atenção primária é a Saúde da Família. É considerado um modelo de atenção primária de saúde focado na unidade familiar e inserido na comunidade 4 .

Estudos internacionais têm evidenciado que a atenção primária tem impacto positivo sobre vários indicadores de saúde; reduz gastos totais; melhora o acesso a serviços de saúde, a qualidade global da atenção à saúde e as ações preventivas; facilita a detecção precoce de problemas reduzindo internações hospitalares; garante cuidados mais apropriados e reduz o uso de cuidados desnecessários de atenção especializada à saúde 5. Estudos no Brasil 6,7,8 apontam na mesma direção: a Saúde da Família melhora o acesso e tem alguns efeitos positivos na situação de saúde. Dois recentes estudos longitudinais também evidenciaram impacto positivo sobre a saúde da população. $\mathrm{O}$ primeiro ${ }^{9}$, evidenciou que um aumento de $10 \%$ na cobertura da Saúde da Família estava associado a uma redução de 4,5\% em taxas de mortalidade principalmente devido à prevenção de mortes por diarréia. O segundo 10 revelou impacto positivo na saúde infantil, reduzindo taxas de mortalidades infantil e neonatal, melhorando o acesso e homogeneizando a cobertura da assistência pré-natal.

Em 2006, a Saúde da Família encontrava-se implementada em 5.106 municípios, cobrindo $46,2 \%$ da população 11 . O processo de implementação tem se dado de forma bastante heterogênea: há municípios onde a Saúde da Família implica extensão e ampliação significativa da atenção primária, enquanto em outros a Saúde da Família encontra uma rede de centros de saúde relativamente consolidados, ainda que freqüentemente com capacidade insuficiente para atender a demanda. Na literatura são apontados vários elementos que distinguem a atenção primária prestada pela Saúde da Família da atenção prestada por tradicionais centros de saúde, entre os quais se destacam: delimitação da área de abrangência com adstrição de clientela, ênfase na ação da equipe da saúde da família em contraposição à ênfase na intervenção médica, organização da demanda, ênfase na atenção integral e enfoque familiar. Entretanto, na prática as diferenças nem sempre são grandes. Centros de saúde possuem na prática uma clientela adscrita, mesmo quando não formalizada; muitas vezes já ampliaram as ações preventivas e o enfoque familiar nunca estava totalmente ausente (por exemplo, Minas Gerais organizava prontuários por família já na década de setenta). Nesse contexto, torna-se importante avaliar as percepções a respeito da atuação de unidades da saúde da família e das tradicionais unidades de saúde e avaliar até que ponto a Saúde da Família atende às expectativas dos usuários, tendo em vista o pressuposto de que estas modelam a atuação dos profissionais de saúde.

Não há uma definição unívoca da atenção primária no contexto da Saúde da Família. Vários autores apontam que a Saúde da Família implica uma conceituação mais ampla da atenção primária. Nessa perspectiva, Andrade et al. 4 (p. 804) a definem "como um modelo de atenção primária, operacionalizado mediante estratégias/ações preventivas, promocionais, de recuperação, reabilitação e cuidados paliativos das equipes de saúde da família, comprometidas com a integralidade da assistência à saúde, focado na unidade familiar e consistente com o contexto socioeconômico, cultural e epidemiológico da comunidade em que está inserido".

Para estudos avaliativos de natureza comparativa, uma definição mais funcional é mais adequada. Nessa perspectiva, este estudo que foi desenvolvido como subprojeto do Desenvolvimento de Estudos Avaliativos - Linha de Base do Projeto de Expansão e Consolidação do Saúde da Família (PROESF) do Ministério da Saúde, tem como referência a definição de Starfield 12 (p. 28): "Aquele nível de um sistema de serviço de saúde que funciona como porta de entrada no sistema, atendendo todas as necessidades e problemas de saúde da pessoa (não direcionada apenas para a enfermidade), ao longo do tempo, fornece atenção para todas as condições, exceto as muito incomuns ou raras, e coordena ou integra os outros tipos de atenção fornecidos em algum outro lugar ou por terceiros". Mesmo referindo-se a um nível do sistema de saúde, essa definição enfoca, antes de tudo, o conjunto de funções ou atribuições da atenção primária. Tem como pressuposto que as 
quatro interpretações de atenção primária discutidas por Vuori 13 estão inter-relacionadas: um conjunto de atividades, um nível de atenção à saúde, uma estratégia para organizar a atenção à saúde e uma filosofia que permeia o sistema de saúde.

A definição de Starfield é congruente com as definições do Institute of Medicine dos Estados Unidos que igualmente tem como referência um conjunto de atribuições. As definições do Institute of Medicine estão subjacentes ao Primary Care Assessment Tool (PCAT), desenvolvido pela John Hopkins School of Public Health, Primary Care Policy Center for Underserved People para avaliar a extensão e a qualidade da atenção primária 14. Em 1978, o Institute of Medicine 15 (p. 16) definiu a atenção primária como "cuidado acessivel, abrangente, coordenado e contínuo, prestado por profissionais responsáveis por serviços de saúde de caráter pessoal". Essa definição contrasta com a da Declaração de Alma-Ata ${ }^{1}$ que inclui ações de saúde pública, e de acordo com o Institute of Medicine é mais apropriada para países que têm maiores necessidades básicas. Considerando o desenvolvimento de sistemas integrados de prestação de serviços de saúde e tendências futuras, o Institute of Medicine 16 (p. 31) apresentou uma nova definição em 1996: “atenção primária é a provisão de serviços de saúde integrados e acessíveis por clínicos que são responsáveis pelo atendimento da grande maioria das necessidades pessoais de serviços de saúde, desenvolvendo uma parceria sustentável com pacientes e atuando no contexto da família e da comunidade". Essa definição continua privilegiando serviços de saúde prestados a pessoas por clínicos, mas inclui três elementos novos: o paciente e a sua família, a comunidade e o sistema integrado de serviços de saúde. Assim, a relação médico/paciente deve ser compreendida no contexto da família e da comunidade e é facilitada e ampliada por equipes de saúde e sistemas integrados de serviços de saúde.

Baseado nessas definições multidimensionais, o PCAT que inclui o Child Consumer/Client Survey, o Adult Consumer/Client Survey e o Facility/Provider Survey, distingue quatro domínios ou atributos principais de atenção primária: primeiro contato, longitudinalidade, abrangência e coordenação. O primeiro contato implica acessibilidade e uso de serviços para cada novo problema ou episódio, para o qual as pessoas procuram cuidados de saúde. A longitudinalidade pressupõe a existência de uma fonte regular de cuidados e seu uso ao longo do tempo. A abrangência (comprehensiveness) implica que serviços de atenção primária devem ser capazes de propiciar todos os tipos de serviços de saúde, incluindo encami- nhamentos a serviços secundários para consultas, serviços terciários para situações específicas, e serviços essenciais de apoio tais como atendimento domiciliar e outros serviços comunitários. A coordenação de cuidados requer alguma forma de continuidade, seja por meio do atendimento dos clínicos, seja por meio de prontuários ou por ambos, como também o reconhecimento de problemas tratados em outros serviços e a integração destes cuidados no acolhimento total do paciente. O PCAT distingue ainda três domínios derivados: enfoque familiar, orientação para a comunidade e competência cultural. O enfoque familiar refere-se ao reconhecimento de fatores familiares relacionados à gênese e tratamento da doença. A orientação para a comunidade diz respeito ao conhecimento dos prestadores sobre as necessidade dos pacientes e o envolvimento na comunidade. A competência cultural refere-se à adaptação dos prestadores no sentido de facilitar relações com populações de caraterísticas culturais específicas 16 .

O PCAT foi adaptado por Macinko et al. 17 para o Brasil, com o desenvolvimento de quatro versões, respectivamente para gestores, profissionais, usuários e acompanhantes de crianças ou pessoas incapacitadas, e com algumas modificações. Em contraste com a versão original, a versão brasileira possui 8 dimensões: acessibilidade, porta de entrada, longitudinalidade, elenco de serviços (comprehensiveness), coordenação (ou integração dos serviços), enfoque familiar, orientação para a comunidade, e formação profissional. As duas primeiras dimensões pertenciam na versão original ao domínio ou atribuição primeiro contato, e a última (formação profissional) é uma dimensão nova em substituição ao domínio da competência cultural, e pressupõe que a atenção primária seja uma área de "especialização" que requer formação específica voltada para o desempenho das funções segundo as dimensões anteriores.

É importante ressaltar que a definição de atenção primária que subjaz o PCAT distinguese em alguns aspectos importantes da definição da atenção primária relacionada à Saúde da Família. Enquanto a primeira enfoca, antes de tudo, a relação médico/paciente, por mais que o relatório do Institute of Medicine de 1996 observe as articulações crescentes e conexões, sempre em mudança, entre saúde pública e cuidados prestados às pessoas, a Saúde da Família enfoca a unidade familiar. Para o Institute of Medicine, o agente principal na atenção primária é o clínico, eventualmente assistido por uma equipe, mas na Saúde da Família o principal agente é a equipe multidisciplinar, o que possibilita a integração entre os cuidados prestados às famílias e ações 
de saúde coletiva em nível local, e a ampliação da prática clínica com a sua dimensão subjetiva e com a inclusão da dimensão social.

O enfoque do PCAT na prestação de serviços pessoais de saúde por clínicos implica limitações quanto ao seu uso para avaliar a atuação de equipes da saúde da família. Pelas dimensões contempladas pelo instrumento, que em quase todas as formas tem como referência o médico clínico, ainda que chamado de profissional, e seus pacientes, este é pouco sensível para os aspectos em que a saúde da família difere da atenção primária preconizada pelo Institute of Medicine, principalmente no que diz respeito às ações dos agentes comunitários e às famílias como foco de atenção.

\section{Metodologia}

O estudo abrange sete municípios do Estado de Goiás (Águas Lindas de Goiás, Anápolis, Aparecida de Goiânia, Goiânia, Luziânia, Rio Verde e Valparaiso de Goiás) e dois de Mato Grosso do Sul (Campo Grande e Dourados). São municípios cuja população varia de aproximadamente 120 mil até 450 mil habitantes, com exceção das duas capitais. A taxa de urbanização encontra-se acima de 90\%. Quatro municípios, incluindo as duas capitais, apresentam um desenvolvimento alto, com Índices de Desenvolvimento Humano (IDH) de 0,80 ou superiores, e quatro outros apresentam IDH entre 0,76 e 0,79, e apenas um município apresenta um grau de desenvolvimento inferior com o IDH de $0,72 \%$ (Tabela 1 ). A cobertura populacional da Saúde da Família variava em 2005 de 7\% em Luziânia a 34,5 \% em Dourados (Tabela 2). Ao longo do período de 2001 a 2005 houve oscilações importantes na cobertura da saúde da família.

Em cada município foram selecionadas por meio de amostragem $20 \%$ das unidades de atenção primária, aplicando-se como critério uma distribuição eqüitativa de unidades com e sem saúde da família, situadas em áreas com maior e menor risco social e em áreas rurais e urbanas. Quando nos municípios menores, 20\% das unidades correspondiam a menos que seis unidades, o número foi completado até seis de acordo com os critérios estipulados. Devido à alta taxa de urbanização o número de unidades selecionadas em áreas rurais foi muito pequeno, não permitindo o uso desta variável na análise dos dados. A amostra total incluiu 36 unidades com saúde da família e 28 unidades básicas tradicionais.

Nas unidades selecionadas foram entrevistados usuários e profissionais de saúde. Os usuários contemplados pelo estudo foram aquelas pesso- as que compareceram à unidade no dia marcado para as entrevistas, buscando qualquer modalidade de atendimento. Para o cálculo da amostra estipulou-se que 40 usuários por dia/unidade formariam o universo. A amostra foi calculada tendo como parâmetros estatísticos um intervalo de confiança igual a $90 \%$ e uma margem de erro de $4 \%$. A aplicação de questionários para os usuários foi realizada em cada unidade de atendimento escolhida no decorrer de um único dia, e a seleção dos entrevistados foi feita por meio de tabela de números aleatórios, usando-se como critério a ordem de chegada/agendamento. No caso de menor de 18 anos, a entrevista foi feita com o/a acompanhante.

Nas unidades selecionadas, todos os profissionais com formação superior foram incluídos. Os com formação média ou elementar participaram da amostra nas seguintes proporções: um profissional por equipe nas unidades com saúde da família, e nas sem este serviço um profissional para cada dois com formação superior. Dessa forma, definiu-se uma amostra de 386 profissionais de nível superior, dos quais 302 foram entrevistados. Previa-se entrevistar 204 pessoas de nível médio ou elementar, mas conseguiu-se entrevistar 207. Quanto aos usuários, estimava-se entrevistar 568, mas foram de fato entrevistados 490 usuários e 133 acompanhantes, perfazendo um total de 623 .

As entrevistas com os profissionais com formação superior foram feitas utilizando-se um questionário auto-aplicável, enquanto os com formação média e elementar e os usuários foram entrevistados mediante a aplicação de questionários por entrevistadores previamente treinados para este fim. Além dos profissionais e usuários dos serviços de saúde, foram entrevistados em cada município o representante dos usuários na mesa diretora do Conselho Municipal de Saúde, o Gestor Municipal do Sistema Único de Saúde (SUS) e o Coordenador da Atenção Básica e/ou Saúde da Família. Desses utilizamos dados apenas quando houve necessidade de aprofundar ou complementar as análises referentes a usuários e a profissionais de saúde.

Do PCAT foram utilizados o questionário para profissionais de saúde, o para usuários dos serviços e o para acompanhantes de crianças e pessoas incapacitadas. Os três questionários abrangem as mesmas dimensões, mas o dos usuários e o dos acompanhantes contêm algumas questões a mais: o questionário dos profissionais é composto de 92 questões, o dos usuários de 107 e o dos acompanhantes de 110 questões. São questões do tipo Likert que possuem seis opções de resposta, às quais foram atribuídos valores numéricos: nunca = 0; quase nunca = 1; algumas 
Tabela 1

Características dos municípios.

\begin{tabular}{|c|c|c|c|c|}
\hline Município & População 2006 & IDH 2000 & $\begin{array}{c}\text { Taxa de crescimento } \\
2000 / 2006(\%)\end{array}$ & $\begin{array}{c}\text { Taxa de urbanização } \\
2000(\%)\end{array}$ \\
\hline Águas Lindas de Goiás & 168.919 & 0,72 & 8,10 & 99,85 \\
\hline Anápolis & 318.807 & 0,79 & 1,70 & 97,25 \\
\hline Aparecida de Goiânia & 453.104 & 0,76 & 5,10 & 99,75 \\
\hline Goiânia & 1.220 .412 & 0,83 & 1,90 & 99,34 \\
\hline Luziânia & 187.263 & 0,76 & 4,80 & 92,26 \\
\hline Rio Verde & 136.231 & 0,8 & 2,60 & 91,01 \\
\hline Valparaiso de Goiás & 123.920 & 0,8 & 4,60 & 100,00 \\
\hline Campo Grande & 765.245 & 0,81 & 2,10 & 98,84 \\
\hline Dourados & 186.357 & 0,79 & 2,40 & 90,89 \\
\hline
\end{tabular}

IDH: Índice de Desenvolvimento Humano.

Fonte: Instituto Brasileiro de Geografia e Estatística. Estimativas populacionais 2006. http://www.ibge.gov.br (acessado em 06/Jun/2006).

Programa das Nações Unidas para o Desenvolvimento. Atlas do desenvolvimento humano. http://www.pnud.org.br/atlas (acessado em 06/Jun/2006).

Tabela 2

Cobertura populacional do saúde da família, 2001-2005.

\begin{tabular}{|c|c|c|c|c|c|}
\hline \multirow[t]{2}{*}{ Município } & \multicolumn{5}{|c|}{ \% população coberta pelo Saúde da Família } \\
\hline & 2001 & 2002 & 2003 & 2004 & 2005 \\
\hline Águas Lindas de Goiás & 27,85 & 27,77 & 26,01 & 24,47 & 15,84 \\
\hline Anápolis & 12,69 & 16,08 & 15,38 & 25,70 & 30,82 \\
\hline Aparecida de Goiânia & 11,45 & 19,97 & 18,34 & 17,39 & 19,05 \\
\hline Goiânia & 18,48 & 21,63 & 18,61 & 17,99 & 17,42 \\
\hline Luziânia & 20,48 & 8,33 & 8,49 & 10,31 & 7,02 \\
\hline Rio Verde & 14,02 & 18,56 & 20,17 & 22,76 & 21,23 \\
\hline Valparaiso de Goiás & 24,31 & 34,49 & 32,22 & 31,16 & 28,82 \\
\hline Campo Grande & 6,67 & 11,34 & 11,55 & 17,36 & 18,33 \\
\hline Dourados & 27,78 & 29,24 & 30,16 & 35,51 & 34,49 \\
\hline
\end{tabular}

Fonte: Ministério da Saúde. Sistema de informação de atenção básica. http://w3.datasus.gov.br/siab.php (acessado em 30/Abr/2006).

vezes $=2 ;$ muitas vezes $=3 ;$ quase sempre $=4 ; \mathrm{e}$ sempre $=5$. Os questionários incluíam também a opção não sabe que na análise foi codificada como valor em branco. Quando $20 \%$ ou mais das respostas se encaixaram nessa categoria, a questão foi excluída.

A média aritmética dos escores obtidos em cada bloco representa o índice composto de cada dimensão de atenção básica, e a média dos oito índices compostos representa o índice composto total da atenção básica. Neste estudo foram calculados os índices compostos para cada dimensão e o índice composto total separadamente para as unidades com e as sem saúde da família. Para avaliar as diferenças foi aplicado o teste-F.
Os dados de usuários e acompanhantes foram analisados em conjunto, uma vez que interessava avaliar o ponto de vista da população atendida.

Os representantes dos usuários na mesa diretora do Conselho Municipal de Saúde foram entrevistados pelos pesquisadores mediante um questionário com perguntas fechadas e abertas que contemplavam as seguintes dimensões do conselho: composição, vínculos de representação, funcionamento e deliberações, participação na formulação das políticas de gestão de recursos humanos e no planejamento orçamentáriofinanceiro, participação nas definições da Saúde da Família e PROESF e avaliação da atuação da Secretaria Municipal de Saúde. Para estudo, fo- 
ram consideradas apenas as respostas referentes aos dois últimos itens. O questionário para os coordenadores de Atenção Básica e/ou Saúde da Família incluía questões sobre a política de formação e contratação de pessoal, atuação da coordenação no planejamento, saúde da família (rotatividade, seleção), práticas de gestão e oferta e avaliação de serviços. Analisamos especificamente as questões referentes à saúde da família sobre rotatividade, formação dos profissionais e satisfação da população. As entrevistas com os gestores, gravadas, eram de natureza semi-estruturada. Foram consideradas as falas a respeito da atenção básica e da Estratégia Saúde da Família.

Este projeto foi aprovado pelo Comitê de Ética da Universidade Federal de Minas Gerais que também aprovou o Termo de Consentimento Esclarecido que foi assinado por todos os entrevistados.

\section{Resultados e discussão}

Os resultados da aplicação do PCAT são apresentados nas Tabelas 3 e 4 . A Tabela 3 apresenta os resultados por dimensão e categoria de entrevistados (usuários, profissionais de níveis médio e superior). A Tabela 4 apresenta os resultados desagregados por município. Os resultados convergem para os que foram obtidos por outros estudos de avaliação da saúde da família que utilizaram o PCAT 18,19,20 , no sentido que apontam para uma melhor avaliação dos serviços prestados pela Estratégia Saúde da Família. Chama, porém, a atenção, que os profissionais de níveis médio e elementar apresentam a melhor avaliação, tanto dos centros tradicionais como da saúde da família, e os usuários a mais baixa. Isso sugere que esses profissionais valorizam mais os serviços públicos de saúde do que os de nível superior.

Analisando os resultados por dimensão, há uma avaliação diferenciada. No caso do acesso, os valores são baixos tanto para os centros de saúde tradicionais como para saúde da família. Chama a atenção que a avaliação da Saúde da Família é mais negativa, ainda que não represente uma diferença significativa. Não se trata apenas de insatisfação de usuários para com o atendimento, pois também os profissionais atribuem uma avaliação baixa ao acesso. Isso sugere que a introdução da saúde da família não implica necessariamente uma melhoria de acesso, mesmo considerando que uma avaliação mais negativa em relação aos centros de saúde poderá resultar do aumento de expectativas. Nesse aspecto, os resultados divergem de outros estudos que sugerem que maiores investimentos na atenção pri- mária aumenta o acesso a serviços de saúde para grupos populacionais de baixa renda 5 .

Tanto os centros de saúde como as equipes da saúde da família funcionam bem como porta entrada. As equipes da saúde da família recebem uma avaliação melhor, mas apenas para os profissionais de nível superior trata-se de uma diferença significativa.

Os resultados sugerem que as equipes da saúde da família estabelecem um vínculo mais forte com os usuários do que os tradicionais centros de saúde. No caso dos profissionais trata-se de uma diferença significativa.

Quanto à modalidade de serviços, os usuários não percebem diferença entre centros de saúde tradicionais e equipes da saúde da família. Entretanto, os profissionais de nível superior atribuem à saúde da família uma melhoria significativa do elenco de serviços ofertados.

Na dimensão coordenação, não há grandes diferenças nem na avaliação de centros de saúde tradicionais e equipes da saúde da família e nem entre usuários e profissionais. O índice composto sugere uma coordenação relativamente adequada.

Quanto ao enfoque familiar e à orientação comunitária, estes estão mais presentes nas equipes da saúde da família do que nos centros de saúde e trata-se de uma diferença significativa. Entretanto, chama a atenção o escore baixo dos usuários. Importante lembrar que os questionários privilegiam a atuação dos profissionais, leia-se dos médicos, e não traduzem a atuação de agentes comunitários. Isso pode explicar o índice composto baixo dos usuários. Os escores bem mais altos dos profissionais podem resultar do fato de que estes incluem na sua avaliação a atuação dos agentes comunitários.

Quanto à formação, há, também, diferença entre as equipes da saúde da família e dos centros tradicionais, embora não seja significativa. As equipes da saúde da família são associadas com melhor formação e orientação.

A Estratégia Saúde da Família foi concebida para expandir o acesso à atenção primária e coordenar a integração com as redes de atenção à saúde: ambulatorial especializada, hospitalar secundária e terciária, rede de serviços de urgência e emergência, e a rede de serviços de atenção à saúde mental e de outros serviços. Em contraste com o esperado, o índice composto da avaliação da dimensão do acesso continua baixo. A baixa avaliação dos usuários é consistente com vários estudos anteriores que apontaram que, mesmo havendo um grau alto de satisfação para com os serviços de saúde, poderá existir insatisfação substancial em relação a componentes específicos da atenção, particularmente o tempo de 
Escores atribuídos às dimensões pelos entrevistados, por categoria e de acordo com tipo de unidade.

\begin{tabular}{|c|c|c|c|c|c|c|}
\hline \multirow[t]{2}{*}{ Dimensões } & \multicolumn{2}{|c|}{$\begin{array}{c}\text { Usuários e } \\
\text { acompanhantes }\end{array}$} & \multicolumn{2}{|c|}{$\begin{array}{l}\text { Profissionais de níveis } \\
\text { médio e elementar }\end{array}$} & \multicolumn{2}{|c|}{$\begin{array}{l}\text { Profissionais de } \\
\text { nível superior }\end{array}$} \\
\hline & ESF & UBS & ESF & UBS & ESF & UBS \\
\hline Acesso & 2,00 & 2,22 & 2,36 & 2,79 & 2,42 & 2,74 \\
\hline Porta de entrada & 4,51 & 4,33 & 4,65 & 4,51 & 4,27 * & 3,82 * \\
\hline Vínculo e responsabilização & 3,92 & 3,63 & 4,30 * & 3,54 * & 4,17 * & 3,46 * \\
\hline Modalidade de serviços & 3,81 & 3,87 & 4,03 & 3,63 & 4,09 * & 3,64 * \\
\hline Coordenação & 3,47 & 3,43 & 3,95 & 3,76 & 3,75 & 3,70 \\
\hline Enfoque familiar & 1,88 * & 1,45 * & 3,89 * & 2,52 * & 3,81 * & 2,83 * \\
\hline Orientação comunitária & 2,23 * & 1,48 * & 3,21 * & 2,13 * & 3,06 * & 2,17 * \\
\hline Profissionais de saúde & 3,74 & 3,64 & 4,09 & 3,75 & 3,87 & 3,52 \\
\hline Índice composto Atenção Básica & 3,19 & 3,01 & 3,81 & 3,33 & 3,68 & 3,24 \\
\hline
\end{tabular}

* $p<0,05$ - valores significativos.

ESF: equipes saúde da família; UBS: unidades básicas de saúde.

Escores atribuídos pelos usuários, profissionais de nível médio e profissionais de nível superior às dimensões da atenção básica segundo tipo de unidade e por município.

\begin{tabular}{|c|c|c|c|c|c|c|c|c|c|c|c|c|c|c|c|c|c|c|}
\hline & \multicolumn{2}{|c|}{ Acesso } & \multicolumn{2}{|c|}{$\begin{array}{l}\text { Porta de } \\
\text { entrada }\end{array}$} & \multicolumn{2}{|c|}{$\begin{array}{l}\text { Vínculo e } \\
\text { responsa- } \\
\text { bilização }\end{array}$} & \multicolumn{2}{|c|}{$\begin{array}{l}\text { Modalidade } \\
\text { de serviços }\end{array}$} & \multicolumn{2}{|c|}{$\begin{array}{l}\text { Coorde- } \\
\text { nação }\end{array}$} & \multicolumn{2}{|c|}{$\begin{array}{l}\text { Enfoque } \\
\text { familiar }\end{array}$} & \multicolumn{2}{|c|}{$\begin{array}{l}\text { Orientação } \\
\text { comunitária }\end{array}$} & \multicolumn{2}{|c|}{ Profissionais } & \multicolumn{2}{|c|}{$\begin{array}{c}\text { Índice de } \\
\text { Atenção } \\
\text { Básica }\end{array}$} \\
\hline & ESF & UBS & ESF & UBS & ESF & UBS & ESF & UBS & ESF & UBS & ESF & UBS & ESF & UBS & ESF & UBS & ESF & UBS \\
\hline \multicolumn{19}{|l|}{ Águas Lindas } \\
\hline \multicolumn{19}{|l|}{ de Goiás } \\
\hline Usuários & 1,7 & 2,0 & 4,3 & 4,4 & 3,8 & 3,6 & 2,6 & 2,6 & 1,5 & 1,4 & 1,2 & 1,2 & 1,0 & 0,6 & 3,3 & 3,3 & 2,4 & 2,4 \\
\hline \multicolumn{19}{|l|}{ Profissionais } \\
\hline de nível médio & 2,4 & 3,3 & 5,0 & 5,0 & 4,3 & 4,0 & 4,1 & 2,5 & 3,7 & 2,9 & 3,6 & 2,8 & 3,1 & 1,6 & 4,2 & 3,6 & 3,8 & 3,2 \\
\hline \multicolumn{19}{|l|}{ Profissionais de } \\
\hline nível superior & 2,3 & 2,8 & 4,0 & 4,7 & 4,1 & 4,1 & 4,0 & 2,9 & 3,8 & 3,1 & 3,4 & 3,1 & 3,0 & 1,5 & 3,6 & 3,6 & 3,5 & 3,2 \\
\hline \multicolumn{19}{|l|}{ Anápolis } \\
\hline Usuários & 2,3 & 2,6 & 4,3 & 4,0 & 4,2 & 3,3 & 2,4 & 2,7 & 2,0 & 1,6 & 1,1 & 1,4 & 1,7 & 1,1 & 3,8 & 3,2 & 2,7 & 2,5 \\
\hline \multicolumn{19}{|l|}{ Profissionais de } \\
\hline nível médio & 2,1 & 2,7 & 4,6 & 4,0 & 4,4 & 3,9 & 3,3 & 2,6 & 3,0 & 2,4 & 2,1 & 1,8 & 1,8 & 0,6 & 3,8 & 3,9 & 3,2 & 2,7 \\
\hline \multicolumn{19}{|l|}{ Profissionais de } \\
\hline nível superior & 2,3 & 2,9 & 3,9 & 3,0 & 4,0 & 3,1 & 4,2 & 3,1 & 3,7 & 3,0 & 3,3 & 2,4 & 3,2 & 1,9 & 4,3 & 3,1 & 3,6 & 2,8 \\
\hline \multicolumn{19}{|l|}{ Aparecida de } \\
\hline \multicolumn{19}{|l|}{ Goiânia } \\
\hline Usuários & 1,7 & 2,0 & 4,5 & 4,0 & 3,7 & 3,7 & 2,7 & 2,5 & 1,6 & 3,0 & 1,7 & 1,6 & 1,0 & 0,6 & 3,2 & 3,4 & 2,5 & 2,6 \\
\hline \multicolumn{19}{|l|}{ Profissionais de } \\
\hline nível médio & 2,2 & 2,8 & 5,0 & 5,0 & 4,1 & 3,4 & 3,6 & 3,1 & 3,5 & 3,4 & 4,6 & 3,2 & 3,6 & 1,3 & 4,4 & 4,0 & 3,9 & 3,3 \\
\hline \multicolumn{19}{|l|}{ Profissionais de } \\
\hline nível superior & 2,0 & 3,0 & 3,8 & 3,3 & 3,8 & 2,8 & 3,7 & 2,9 & 3,2 & 3,1 & 4,7 & 2,6 & 2,4 & 1,5 & 3,9 & 4,2 & 3,4 & 2,9 \\
\hline
\end{tabular}

(continua) 


\begin{tabular}{|c|c|c|c|c|c|c|c|c|c|c|c|c|c|c|c|c|c|c|}
\hline & \multicolumn{2}{|c|}{ Acesso } & \multicolumn{2}{|c|}{$\begin{array}{l}\text { Porta de } \\
\text { entrada }\end{array}$} & \multicolumn{2}{|c|}{$\begin{array}{l}\text { Vínculo e } \\
\text { responsa- } \\
\text { bilização }\end{array}$} & \multicolumn{2}{|c|}{$\begin{array}{l}\text { Modalidade } \\
\text { de serviços }\end{array}$} & \multicolumn{2}{|c|}{$\begin{array}{l}\text { Coorde- } \\
\text { nação }\end{array}$} & \multicolumn{2}{|c|}{$\begin{array}{l}\text { Enfoque } \\
\text { familiar }\end{array}$} & \multicolumn{2}{|c|}{$\begin{array}{l}\text { Orientação } \\
\text { comunitária }\end{array}$} & \multicolumn{2}{|c|}{ Profissionais } & \multicolumn{2}{|c|}{$\begin{array}{c}\text { Índice de } \\
\text { Atenção } \\
\text { Básica }\end{array}$} \\
\hline & ESF & UBS & ESF & UBS & ESF & UBS & ESF & UBS & ESF & UBS & ESF & UBS & ESF & UBS & ESF & UBS & ESF & UBS \\
\hline \multicolumn{19}{|l|}{ Goiânia } \\
\hline Usuários & 2,1 & 2,4 & 4,5 & 4,2 & 3,7 & 3,5 & 2,7 & 2,8 & 2,7 & 2,2 & 2,1 & 1,4 & 1,7 & 0,7 & 3,4 & 3,4 & 2,8 & 2,6 \\
\hline \multicolumn{19}{|l|}{ Profissionais de } \\
\hline nível médio & 2,0 & 2,6 & 4,5 & 4,6 & 4,0 & 3,1 & 3,6 & 3,4 & 3,6 & 3,6 & 3,9 & 2,3 & 2,8 & 2,3 & 4,3 & 4,0 & 3,6 & 3,2 \\
\hline \multicolumn{19}{|l|}{ Profissionais de } \\
\hline nível superior & 2,4 & 2,9 & 4,5 & 3,6 & 3,9 & 3,2 & 3,8 & 3,2 & 3,6 & 3,3 & 4,0 & 2,5 & 2,8 & 1,8 & 3,9 & 3,5 & 3,6 & 3,0 \\
\hline \multicolumn{19}{|l|}{ Luziânia } \\
\hline Usuários & 1,5 & 1,8 & 4,2 & 4,6 & 3,9 & 3,6 & 2,7 & 2,7 & 1,8 & 1,8 & 1,6 & 1,0 & 1,0 & 0,9 & 3,2 & 3,2 & 2,5 & 2,5 \\
\hline \multicolumn{19}{|l|}{ Profissionais de } \\
\hline nível médio & 2,2 & 2,1 & 5,0 & 5,0 & 4,2 & 3,7 & 3,6 & 2,8 & 3,8 & 3,0 & 4,3 & 2,5 & 2,2 & 0,9 & 5,0 & 3,4 & 3,8 & 2,9 \\
\hline \multicolumn{19}{|l|}{ Profissionais de } \\
\hline nível superior & 2,4 & 2,3 & 3,6 & 4,0 & 4,0 & 3,2 & 3,7 & 2,8 & 3,3 & 3,1 & 4,1 & 2,7 & 2,0 & 0,7 & 4,4 & 3,4 & 3,4 & 2,8 \\
\hline \multicolumn{19}{|l|}{ Rio Verde } \\
\hline Usuários & 1,8 & 1,7 & 4,6 & 4,0 & 3,6 & 3,3 & 3,0 & 2,6 & 2,0 & 1,6 & 2,9 & 0,8 & 1,5 & 0,5 & 3,9 & 2,8 & 2,8 & 2,2 \\
\hline \multicolumn{19}{|l|}{ Profissionais de } \\
\hline nível médio & 2,4 & 1,7 & 2,8 & 4,5 & 3,8 & 2,6 & 4,8 & 3,0 & 2,2 & 1,1 & 4,5 & 1,6 & 2,6 & 0,3 & 2,9 & 3,5 & 3,3 & 2,3 \\
\hline \multicolumn{19}{|l|}{ Profissionais de } \\
\hline nível superior & 2,3 & 2,3 & 4,2 & 2,7 & 3,9 & 2,9 & 4,1 & 2,9 & 3,5 & 3,2 & 4,1 & 2,2 & 3,8 & 1,2 & 4,7 & 3,0 & 3,8 & 2,5 \\
\hline \multicolumn{19}{|l|}{ Valparaiso } \\
\hline Usuários & 2,0 & 2,7 & 4,2 & 4,4 & 3,9 & 3,7 & 3,6 & 4,3 & 3,3 & 3,5 & 1,8 & 1,8 & 1,6 & 1,7 & 3,6 & 3,7 & 3,0 & 3,2 \\
\hline \multicolumn{19}{|l|}{ Profissionais de } \\
\hline nível médio & 2,0 & 4,3 & 2,8 & 3,0 & 4,6 & 3,9 & 3,6 & 4,1 & 3,8 & 3,2 & 3,3 & 2,1 & 3,2 & 2,1 & 3,5 & 3,1 & 3,4 & 3,3 \\
\hline \multicolumn{19}{|l|}{ Profissionais de } \\
\hline nível superior & 2,3 & 4,3 & 3,9 & 4,3 & 4,0 & 3,6 & 3,6 & 3,8 & 3,2 & 3,6 & 2,9 & 2,7 & 3,1 & 2,2 & 2,9 & 2,8 & 3,2 & 3,4 \\
\hline Campo Grande & & & & & & & & & & & & & & & & & & \\
\hline Usuários & 2,5 & 2,3 & 4,4 & 4,4 & 4,1 & 3,5 & 3,3 & 3,1 & 3,1 & 2,9 & 2,4 & 1,9 & 2,4 & 2,4 & 4,1 & 3,8 & 3,3 & 3,0 \\
\hline Profissionais de & & & & & & & & & & & & & & & & & & \\
\hline nível médio & 2,5 & 2,5 & 4,6 & 4,1 & 4,2 & 3,6 & 4,1 & 3,7 & 3,8 & 3,9 & 3,2 & 2,5 & 3,5 & 2,4 & 4,1 & 3,9 & 3,7 & 3,4 \\
\hline Profissionais de & & & & & & & & & & & & & & & & & & \\
\hline nível superior & 2,7 & 2,7 & 4,8 & 4,4 & 4,2 & 3,2 & 4,2 & 3,6 & 3,7 & 3,7 & 3,2 & 2,5 & 3,3 & 2,8 & 3,7 & 3,7 & 3,7 & 3,3 \\
\hline Dourados & & & & & & & & & & & & & & & & & & \\
\hline Usuários & 2,1 & 2,2 & 4,8 & 4,0 & 3,6 & 3,7 & 2,9 & 2,8 & 2,2 & 2,3 & 0,9 & 0,2 & 2,0 & 1,2 & 3,7 & 3,8 & 2,8 & 2,5 \\
\hline Profissionais de & & & & & & & & & & & & & & & & & & \\
\hline nível médio & 2,2 & 3,0 & 5,0 & 5,0 & 4,5 & 3,2 & 4,6 & 4,0 & 3,0 & 4,2 & 4,7 & 2,6 & 4,6 & 3,1 & 3,9 & 3,4 & 4,1 & 3,6 \\
\hline Profissionais de & & & & & & & & & & & & & & & & & & \\
\hline nível superior & 2,5 & 2,6 & 4,1 & 3,2 & 4,3 & 2,6 & 3,9 & 3,1 & 3,8 & 2,8 & 4,2 & 1,9 & 3,4 & 1,9 & 3,5 & 3,5 & 3,7 & 2,7 \\
\hline
\end{tabular}

ESF: equipes saúde da família; UBS: unidades básicas de saúde.

espera e a comunicação entre profissionais e usuários 20 .

Desagregando os dados por município (Tabela 4), observa-se que há variações dentro do panorama geral. Com pequenas exceções, o índice composto total de atenção básica é mais favorável para as equipes da saúde da família em todos os municípios, e a avaliação dos profissionais é sempre mais favorável. Nos municípios com menor IDH, Águas Lindas de Goiás, Aparecida de
Goiânia e Luziânia, que têm maior crescimento populacional, índices compostos referentes ao acesso são inferiores para ambos os tipos de unidades. Isso poderá ser resultado do excesso da demanda por serviços de saúde, que ainda é maior no caso de unidades com Saúde da Família, uma vez que quase sempre as equipes são implantadas em bairros com baixas condições socioeconômicas. Além disso, deve ser considerado que esses municípios estão sendo confron- 
tados com a necessidade de organizar o sistema de saúde como um todo, incluindo a organização de hospitais públicos e o acesso a procedimentos de média complexidade. Nessas condições, a valorização da Saúde da Família tende a ser menor. Num desses municípios o Secretário afirma que a "Saúde da Família é cara para o município e que o impacto é pequeno, pois o Programa Saúde da Família (PSF) vira posto". Ao mesmo tempo, um representante do Conselho Municipal de Saúde opina: "Acho que o município adotou o PSF para receber recursos da União. Em alguns bairros dá certo, em outros não. A preocupação maior é atingir metas e não o usuário. Não tem padrão de atendimento". Para essa situação, são significativos os valores muito baixos do índice composto de orientação comunitária e do enfoque familiar, o que possivelmente está relacionado também com a falta de oportunidades de cursos e treinamentos para as equipes da saúde da família. $\mathrm{Na}$ maioria das vezes o profissional novo faz apenas um treinamento introdutório ou um treinamento em serviços, a despeito do fato de que a saúde da família exige uma reorientação das práticas profissionais.

A insatisfação dos usuários com o acesso aos serviços transparece também nas falas dos conselheiros de saúde. Mesmo fazendo comentários favoráveis ("excelente como proposta de atenção básica; foi criada com objetivo de resgatar cidadania; diagnósticos preventivos contribuíram para diminuir os agravos decorrentes das doenças”) expressam críticas: "[a Saúde da Família] não cumpre o papel a contento; baixa cobertura; pouca resolutividade; virou uma substituição do déficit da rede; dificuldade de constituição da equipe; mais equipes são necessárias; as equipes estão ficando nos postos; não se faz mais visitas como no início".

Em contraste com os conselheiros de saúde, os coordenadores da atenção básica, quase sempre, afirmam que os usuários estão satisfeitos. Mas é significativo que, ao mesmo tempo, eles apontam para as condições que poderão dificultar o acesso e gerar insatisfação: falta de recursos humanos e falta de condições físicas.

É apenas a falta que explica a reprodução de práticas tradicionais? É o PCAT que não nos permite colocar em evidência as inovações, ou a prática médica tradicional resiste bravamente a mudanças?

\section{Considerações finais}

Salta aos olhos que há uma diferença nítida entre a avaliação dos usuários e a dos profissionais de saúde. Para quase todas as dimensões a avalia- ção dos usuários é menos positiva e, além disto, ela difere pouco quando comparada às unidades com e sem saúde da família. Apenas para as dimensões enfoque familiar e orientação comunitária observa-se uma diferença estatisticamente significativa em favor das unidades com saúde da família. Trata-se de valores baixos e aquém do esperado considerando os princípios e as diretrizes da Saúde da Família. Entretanto, há de se considerar que o PCAT mensura a ação dos profissionais (na maioria dos casos os médicos e em alguns momentos incluindo também enfermeiras) e não das equipes com inclusão dos agentes comunitários. $\mathrm{O}$ fato de que o acesso no caso das unidades sem saúde da família é mais bem avaliado pelas três categorias de respondentes, sugere que as equipes saúde da família nos municípios em tela não estão obtendo resultados maiores que as unidades tradicionais. Isso significa que provavelmente ainda se está longe de produzir uma reorientação da atenção primária, tal como preconizada pelo discurso oficial, mesmo considerando que as unidades tradicionais possam caminhar na direção proposta. Ainda que este resultado não possa ser generalizado, pois o estudo diz respeito apenas às cidades de mais de 100 mil habitantes nos Estados de Goiás e Mato Grosso do Sul, e que nem podemos tratar cada município como um estudo de caso devido à amostra reduzida em cada lugar, ele suscita várias indagações, particularmente pelo fato de que outros estudos avaliativos sobre a Saúde da Família encontraram resultados semelhantes.

A dimensão do acesso diz respeito principalmente à maior ou menor facilidade da população para obter uma consulta. Ainda que não devamos reduzir a atenção primária à prestação de consultas médicas, esta atenção não pode abdicar da atenção clínica prestada pelos médicos, ou de acordo com os problemas apresentados por outros profissionais tais como enfermeiros e psicólogos, mas têm de integrá-la à vertente da Saúde Coletiva. O fato de que as equipes da Saúde da Família não conseguem avançar nessa perspectiva pode ser resultado de uma estratégia ainda em construção. Entretanto, a dificuldade de estratégias de organização ou reorientação da atenção básica em obter os resultados almejados, poderá apontar para problemas sobre os quais a Estratégia Saúde da Família não consegue atuar.

Um desses problemas é a forma de inserção do médico no mercado de trabalho e nos serviços públicos de saúde, que tende a influir também sobre a inclusão de outros profissionais de nível superior. O que mais marca essa inserção é a jornada de quatro horas, conquistada pela categoria no início da década de sessenta, que tem como resultado a multiplicação de empregos e 
locais de trabalho, fazendo com que os médicos e eventualmente outros profissionais deixem de se integrar nas equipes de trabalho. A Saúde da Família insiste em horário integral de acordo com seu discurso, mas, principalmente em cidades maiores, isto resulta em alta rotatividade dos médicos e não raras vezes na falta destes. Esse problema não se resolve meramente por meio de uma política que o ignora. Rever a jornada de trabalho de quatro horas do médico é impossível a curto e médio prazos e dependerá não apenas de iniciativas do SUS, mas do envolvimento do setor privado, ou seja, o mercado como um todo. Em face dessa situação, há de se encontrar a curto prazo modalidades diferenciadas para os médicos e outros profissionais de nível superior.

Quanto à reorientação da atenção primária pela Saúde da Família, não podemos deixar de considerar que transformações de sistemas de saúde em grande parte se realizam como conseqüência não apenas de políticas de saúde, mas de processos relativamente autônomos, tais como a racionalização funcional da atenção à saúde e a mudanças de concepções dominantes sobre os processos de saúde/doença e a atenção à saúde. Quanto à racionalização da atenção à saúde, há de se destacar que esta passou por um processo de divisão funcional como resultado de um processo de divisão de trabalho e especialização, com maior impacto na área da clínica devido ao desenvolvimento da tecnologia médica. Os profissionais perseguem, grosso modo, dois objetivos: seu próprio bem-estar, em geral resultado de renda, legitimidade e prestígio de suas atividades profissionais; e o bem-estar da população. A despeito dos esforços de valorizar as equipes de saúde da família por meio de cursos de qualificação, congressos e prêmios, o médico da equipe da saúde da família não é socialmente valorizado entre seus pares onde a valorização é medida pelo uso de tecnologias cada vez mais avançadas.

A racionalização invade também a vida cotidiana, fazendo com que crescentemente a população defina seus problemas de saúde em termos emprestados dos discursos profissionais. Devido ao crescente uso de tecnologias médicas e insumos tais como medicamentos, a interação entre profissionais e usuários é objeto de terceiros (indústrias de insumos etc.) que na perseguição de seus interesses contribuem para reprodução duma relação entre médicos e usuários em que a tecnologia se torna mediadora.

As limitações deste modo de atuar pressionaram para mudanças dos discursos e práticas da saúde, redefinindo o objeto de intervenção no nível da atenção básica: a pessoa, família ou grupos que almejam qualidade de vida, superação de riscos de adoecimento e orientação quando entram na rede da tecnologia médica. Como esta tendência vai se expressar no sistema de saúde, dependerá da criação de novas oportunidades de realização para os profissionais, mas não apenas isto. Implica também a modificação das expectativas dos usuários. Nesse contexto, a Saúde da Família parece uma estratégia demasiadamente tímida, quanto à antecipação de mudanças, pois aposta muito em objetivos amplos e no papel de agentes comunitários e pouco coloca em questão as relações de trabalho dentro das equipes, condicionadas por longos anos de formação em direções não compatíveis com as propostas e pela forte presença da tecnológica biomédica.

\section{Resumo}

Este estudo trata da percepção do desempenho de unidades básicas de saúde com e sem Saúde da Família em cidades com mais de 100 mil habitantes em Goiás e Mato Grosso do Sul. Foram aplicados os questionários do Primary Care Assessment Tool (PCAT) elaborado pela John Hopkins University e adaptado para o Brasil, que contempla oito dimensões de atenção básica, a uma amostra de 36 unidades básicas com Saúde da Família e 28 unidades tradicionais, onde foram entrevistados 302 profissionais de nível superior, 207 de nível médio, 490 usuários e 133 acompanhantes. Os dados não apresentaram diferenças significativas entre as unidades com e sem saúde da família, mas as per- cepções dos profissionais são sempre mais favoráveis em comparação às dos usuários. Analisando os dados por dimensão, observou-se que para todas as dimensões os "dados das unidades com saúde da família" são mais favoráveis com exceção da "dimensão do acesso". Entretanto, apenas para as dimensões de enfoque familiar e orientação comunitária a diferença entre a avaliação de unidades com e sem saúde da família é significativa para todas as três categorias.

Atenção Primária à Saúde; Saúde da Família; Avaliação; Percepção 


\section{Colaboradores}

C. J. van Stralen foi responsável pela concepção e redação do artigo. S. A. Belisário coordenou o estudo avaliativo e participou da coleta, análise e discussão dos resultados. T. B. S. van Stralen e A. M. D. Lima desenvolveram o estudo avaliativo e participaram da coleta, sistematização e análise de dados e da revisão final do artigo. A. W. Massote colaborou na sistematização dos dados do estudo avaliativo. C. L. Oliveira foi responsável pelo tratamento estatístico dos dados.

\section{Referências}

1. World Health Organization. Declaration of AlmaAta: International Conference on Primary Health Care. Geneva: World Health Organization; 1978.

2. Brasil. Decreto no. 78.307. Aprova o Programa de Interiorização das Ações de Saúde e Saneamento no Nordeste e dá outras providências. Diário Oficial da União 1976; 25 ago.

3. Seixas JC. Tema III Interiorização dos Serviços de Saúde. In: Ministério da Saúde, organizador. VI Conferência Nacional de Saúde; 1977. http://bvs ms.saude.gov.br/bvs/publicacoes/0216cns_te ma3.pdf (acessado em 05/Jun/2007).

4. Andrade LOM, Barreto ICHC, Bezerra.RC. Atenção primária à saúde e estratégia saúde da família. In: Campos GWS, Minayo MCS, Akerman M, Drumond Jr. M, Carvalho, YM, organizadores. Tratado de saúde coletiva. São Paulo: Editora Hucitec/Rio de Janeiro: Editora Fiocruz; 2006. p. 783-836.

5. Starfield B, Shi L, Macinko J. Contribution of primary care to health systems and health. Milbank Q 2005; 83:457-502.

6. Araújo MR, Assunção RS. The practice of the community health agency in health promotion and disease prevention. Rev Bras Enferm 2004; 57:19-25.

7. Conill EM. Políticas de atenção primária e reformas sanitárias: discutindo a avaliação a partir da análise do Programa Saúde da Família em Florianópolis, Santa Catarina, Brasil, 1994-2000. Cad Saúde Pública 2002; 18 Suppl:S191-202.

8. Moura ER, Holanda FJ, Rodrigues, MS. Avaliação da assistência pré-natal oferecida em uma microrregião de saúde do Ceará, Brasil. Cad Saúde Pública 2003; 19:1791-9.

9. Macinko J, Guanais FC, Souza MFM. Evaluation of the impact of the Family Health Program on infant mortality in Brazil, 1990-2002. J Epidemiol Community Health 2006; 60:13-20.

10. Bezerra RCR. Evaluation of the impact of a national primary health care policy on infant health outcomes from 1999-2002 [Tese de Doutorado]. Tucson: Mel \& Enid Zuckermann College of Public Health, University of Arizona; 2006.
11. Departamento de Atenção Básica, Ministério da Saúde. Saúde da família. http://dtr2004.saude.gov. br/dab/ (acessado em 26/Jun/2007).

12. Starfield B. Atenção primária: equilíbrio entre necessidades de saúde, serviços e tecnologia. Brasília: Organização das Nações Unidas para a Educação, a Ciência e a Cultura/Ministério da Saúde; 2002.

13. Vuori H. The role of schools of public health in the development of primary health care. Health Policy 1985; 4:221-30.

14. Shi L, Starfield B, Xu J. Validating the adult Primary Care Assessment Tool. J Fam Pract 2001; 50:161-75

15. Institute of Medicine. A manpower policy for primary health care. Washington DC: National Academy of Sciences; 1978. (IOM Publication, 78-02).

16. Institute of Medicine. Primary care: America's health in a new era. Washington DC: National Academy Press; 1996.

17. Macinko J, Almeida C, Oliveira ES, Sá PK, Organization and delivery of primary health care services in Petrópolis, Brazil. Int J Health Plann Manage 2004; 19:303-17.

18. Almeida C, Macinko J. Validação de uma metodologia de uma avaliação rápida das características organizacionais e do desempenho do serviço de atenção básica do Sistema Único de Saúde (SUS) em nível local. Brasília: Organização Pan-Americana da Saúde/Organização Mundial da Saúde/Ministério da Saúde; 2006.

19. Elias PE, Ferreira CW, Alves MCG, Cohn A, Kishima V, Gomes A, et al. Atenção básica em saúde; comparação entre PSF e UBS por estrato de exclusão social em São Paulo. Ciênc Saúde Coletiva 2006; 11:633-41.

20. Ibañez N, Rocha JSY, Viana ALA, Castro PC, Ribeiro MCSA, Forster AC, et al. Avaliação do desempenho da atenção básica no Estado de São Paulo. Ciênc Saúde Coletiva 2006; 11:683-703.

21. Williams SJ, Calnan M. Key Determinants of consumer satisfaction with general practice. J Fam Pract 1991; 8:237-42.

Recebido em 11/Jul/2007

Versão final reapresentada em 28/Nov/2007

Aprovado em 13/Dez/2007 